\title{
Topology change of level sets in Morse theory
}

\author{
Andreas Knauf and Nikolay Martynchuk
}

\begin{abstract}
The classical Morse theory proceeds by considering sublevel sets $f^{-1}(-\infty, a]$ of a Morse function $f: M \rightarrow \mathbb{R}$, where $M$ is a smooth finite-dimensional manifold. In this paper, we study the topology of the level sets $f^{-1}(a)$ and give conditions under which the topology of $f^{-1}(a)$ changes when passing a critical value. We show that for a general class of functions, which includes all exhaustive Morse functions, the topology of a regular level $f^{-1}(a)$ always changes when passing a single critical point, unless the index of the critical point is half the dimension of the manifold $M$. When $f$ is a natural Hamiltonian on a cotangent bundle, we obtain more precise results in terms of the topology of the base space. (Counter-)examples and applications to celestial mechanics are also discussed.
\end{abstract}

\section{Introduction and notation}

Let $M$ be a smooth $m$-dimensional manifold without boundary (in this paper, we consider only separable and metrizable manifolds). We recall that a function $f \in C^{2}(M, \mathbb{R})$ is called a Morse function if for every critical point $x \in M$ of $f$, the Hessian

$$
\operatorname{Hess} f(x): T_{x} M \times T_{x} M \longrightarrow \mathbb{R}
$$

is non-degenerate. One defines the index of a critical point by index $(f, x):=\operatorname{dim}(V)$, where $V \subseteq T_{x} M$ is a subspace of maximal dimension on which $\operatorname{Hess} f(x)$ is negative definite.

The classical Morse theory (see [24] and [27] for background material) proceeds by considering sublevel sets

$$
M^{b}:=\{x \in M \mid f(x) \leq b\} \quad \text { and } \quad M_{a}^{b}:=\{x \in M \mid a \leq f(x) \leq b\} .
$$

Key words and phrases: invariant manifolds, Hamiltonian and celestial mechanics, Morse theory, surgery theory, vector bundles. $70 \mathrm{H} 33$.

2010 Mathematics Subject Classification: 37N05, 55R25, 57N65, 57R65, 58E05, 70F10, 
Under standard compactness assumptions (for instance, if all the sets $M_{a}^{b}$ are compact; see Section 2), for regular values $a<b$ of $f$, the sublevel sets $M^{a}$ and $M^{b}$ are diffeomorphic if $M_{a}^{b}$ contains no critical points. If there is one critical point $x$ in the interior of $M_{a}^{b}$, then $M^{b} \cong M^{a} \cup H_{k}^{m}$, with the handle $H_{k}^{m}:=D^{k} \times D^{m-k}$ of index $k:=\operatorname{index}(f, x)$ attached along $\partial H_{k}^{m}=S^{k-1} \times D^{m-k}$. We note that by the construction, the intersection $M^{a} \cap H_{k}^{m}$ is the subset $S^{k-1} \times D^{m-k} \subset \partial M^{a}$. In particular, $M^{b}$ is homotopy equivalent to the space $M^{a} \cup D^{k}$ obtained from $M^{a}$ by attaching a $k$-cell $D^{k}$. This is implies that $M^{b}$ is not homotopy equivalent to $M^{a}$ if $M$ is compact; cf. (2.1) below.

In the present paper we are interested in the topology of the level sets $f^{-1}(a)=$ $\partial M^{a}$. First we note that $\partial M^{b}$ and $\partial M^{a}$ are always diffeomorphic if $M_{a}^{b}$ contains no critical points. It is not difficult to see that the same can happen if $M_{a}^{b}$ contains several critical points. In fact, simple examples (like Example 2.1 below) show that for regular values $a<b, \partial M^{b}$ may be diffeomorphic to $\partial M^{a}$ even when $M_{a}^{b}$ contains a single critical point. This leads to the following natural question: under which conditions does the topology of $\partial M^{a}$ change (in the rough sense that $H_{\ell}\left(\partial M^{b} ; G\right) \neq$ $H_{\ell}\left(\partial M^{a} ; G\right)$ for some abelian group $G$ and some $\left.\ell \in \mathbb{N}_{0}\right)$, when the function $f$ passes a critical level with one or several critical points? The main goal of the present paper is to develop criteria to answer this question in many cases.

We note that the above topology change question is not completely new. It can be traced back to Maxwell's paper on Hills and Dales [21] published in 1870. It is also very natural in the context of surgery theory, which according to [31] "studies the possible handle structures on manifolds and cobordisms". In particular, the knot complement theorem [13], which states that non-trivial Dehn surgeries on nontrivial knots in $S^{3}$ do not give $S^{3}$, and the so-called cosmetic surgery conjecture [5] and [17], which is a generalisation of the knot complement theorem, are very similar to this question. Recently, it has been raised by A. Albouy in connection with the $n$-body problem and the topology of the corresponding integral manifolds [2], [32] and [33]. The main question in this context, which motivated our work, is whether for the $n$-body problem, the topology of the integral manifolds always changes when passing through a bifurcation level. We note that this is true for the two levels of the Kepler problem with a given nonzero value of angular momentum, for $n=3$ celestial bodies [23], and for the planar $n$-body problem, provided that the reduced Hamiltonian is a Morse function having at most two critical points on each level set; see Section 4 and [22].

To conclude the introduction, we now give an outline of this work together with a short summary of the results.

In Section 2, we consider level sets of abstract Morse functions, which satisfy the so-called Palais-Smale condition [28] and for which the level sets have finitely 
generated homology groups; see Assumptions 2.2. We show that for such a function $f$, the topology of $f^{-1}(a)$ changes when passing a single critical point, if the index $k$ of the critical point is different from $m / 2$, where $m$ is the dimension of the manifold $M$. We also consider the case of several critical points on a given critical level and prove the topology change under a certain assumption on the indices of these critical points. Specifically, it turns out that the topology always changes when passing a given critical level if it contains a critical point of index $k \neq m / 2$ such that there exists no other critical point of index $k-1, k+1$ or $m-k$; see Theorem 2.9. We note that these results apply to functions which do not have to be everywhere Morse (it is sufficient to assume non-degeneracy in a small neighborhood of a given critical level), and the level sets do not have to be compact.

The general discussion of Section 2 is based on the assumption $\left(^{1}\right)$ that there exists a critical point of index $k \neq m / 2$, where $m$ is the dimension of the ambient manifold $M$. And this is to be expected, since to such a critical point there corresponds a surgery on a level set that removes an embedded sphere of dimension $k-1$ and replaces it with a sphere of a different dimension $m-k-1$. When $k=m / 2$ (note that this is always the case in knot theory, for then $k=4 / 2$ ), the spheres have the same dimension and, at least on the level of Morse function on abstract manifolds, both outcomes for the topology change are possible. In fact, it is even possible for a regular level set to bifurcate to another differentiable manifold without changing its homeomorphism type, as examples of exotic spheres show; see Remark 3.9. Nonetheless, it is natural to ask for criteria which guarantee a topology change also in this case of a middle dimension. We address this question in Section 3 for a special class of Morse functions.

More specifically, in Section 3, we consider the case when the manifold $M$ is a rank $n$ vector bundle over an $n$-manifold $N$ and the Morse function $f$ is (up to translations) a sum of a fiberwise positive definite quadratic form and a 'potential' function, which is constant on the fibers. The most important such case is the one of a Hamiltonian function

$$
H: M=T^{*} N \longrightarrow \mathbb{R}, \quad H(q, p)=K(p-A(q))+V(q)
$$

on the cotangent bundle of a base manifold $N$; here $K$ and $V$ are the system's kinetic and potential energy, and $A$ is the magnetic potential. In this case, we show that the topology of $H^{-1}(h)$ always changes when passing a single critical point if the Euler characteristic of the base space $N$ is different from \pm 1 . In the case of abstract vector bundles, we obtain a similar result in terms of the Euler number. We note that the Euler number plays an important role also in the context of classification

$\left({ }^{1}\right)$ We note that a related condition $k<m / 2$ appears very classically in a procedure of killing the homotopy groups of manifolds [16] and [25]. 
of integrable Hamiltonian systems with two degrees of freedom [4] and [12] and monodromy of such systems [10], [19] and [20]. In Section 3 we also consider the special case when $N$ is an $n$-sphere. Because of Adams' result on the Hopf invariant one problem [1], it follows that in this case, no topology change is possible only in dimensions $n=2,4$ and 8 .

We remark that the results of Section 3 indicate that the question of the topology change that we consider in this paper is not only a question about surgeries on abstract level sets; at least for the case of natural Hamiltonians on cotangent bundles, it is intimately related to the global topology of the ambient manifold $M=T^{*} N$ on which the Morse function is defined; see Theorem 3.6 and Corollary 3.8.

In Section 4, we illustrate the developed theory on specific examples from Hamiltonian and celestial mechanics: on the quadratic spherical pendulum, the restricted three-body problem, and the planar $n$-body problem. In particular, we demonstrate that there is always a topology change for the planar $n$-body problem, provided that the reduced Hamiltonian is Morse having at most two critical points on each level set; cf [22].

We conclude the paper with the Appendix, where details of some proofs and a few miscellaneous results are given.

\section{Level sets of Morse functions}

Let $f \in C^{2}(M, \mathbb{R})$ be a Morse function on a manifold $M$ and let $m:=\operatorname{dim}(M)>0$ denote the dimension of this manifold. It is not difficult to see that two level sets $f^{-1}(a)$ and $f^{-1}(b)$ may be diffeomorphic even if there are critical values in the subinterval $[a, b]$. Indeed, one can take a closed manifold $M$ and a Morse function $f$ on this manifold that has unique global minimum and maximum points $x_{\min }$ and $x_{\max }$; then $f^{-1}\left(f\left(x_{\max }\right)-\varepsilon\right)$ and $f^{-1}\left(f\left(x_{\min }\right)+\varepsilon\right)$ are diffeomorphic to a sphere $S^{m-1}$.

This is also possible if between the levels there is only one critical level with a single critical point:

Example 2.1. (No topology change of level sets) Consider the Morse function $f=\tilde{f} \circ \pi^{-1}: \mathbb{R P}(2) \rightarrow \mathbb{R}$, induced by

$$
\tilde{f}: S^{2} \subseteq \mathbb{R}^{3} \longrightarrow \mathbb{R}, \quad \tilde{f}(x)=\sum_{k=1}^{3} k\left|x_{k}\right|^{2},
$$

with projection $\pi: S^{2} \rightarrow \mathbb{R P}(2) \cong S^{2} / S^{0}$. Then $f^{-1}(2)$ is a critical level with the unique critical point $\pm\left(\begin{array}{lll}0 & 1 & 0\end{array}\right)$, and $f^{-1}(2+\varepsilon) \cong S^{1} \cong f^{-1}(2-\varepsilon)$. Note that a similar phenomenon arises for a perfect Morse function $f$ on the complex projective space 
$\mathbb{C P}(2) \cong S^{5} / S^{1}$, with regular level sets diffeomorphic to the sphere $S^{3}$. So it is not caused by lack of orientability.

More generally, there is no topology change for $m$ even at the level of the index $m / 2$ critical point of the Morse function $f=\sum_{k=1}^{m+1} k\left|x_{k}\right|^{2}$ on $\mathbb{R P}(m)$ and, similarly, at the level of the index $m$ critical point of $f=\sum_{k=1}^{m+1} k\left|z_{k}\right|^{2}$ on $\mathbb{C P}(m) \cong S^{2 m+1} / S^{1}$ ( $m$ is assumed even in both cases).

So we consider the following general question: How does the homology of level sets of a Morse function $f: M \rightarrow \mathbb{R}$ on an $m$-dimensional manifold $M$ change when passing a critical value $c \in \mathbb{R}$ ?

First, consider the more usual case of sublevel sets (1.1) and assume, for the moment, that $f$ is exhaustive, that is, that all the sublevel sets

$$
M^{a}=f^{-1}(-\infty, a]
$$

are compact. Consider $a<c<b$ such that there is only one critical point $x_{c} \in f^{-1}(c)$ of $f$ in $M_{a}^{b}$. For $k:=\operatorname{index}\left(f, x_{c}\right)$,

1. $M^{b}\left(M_{a}^{b}\right)$ is homotopy equivalent to $M^{a}$ (respectively, $\partial M^{a}$ ) with a $k$-cell $D^{k}$ attached.

2. $M^{b}$ is diffeomorphic to $M^{a}$ with an $m$-dimensional handle $H_{k}^{m}:=D^{k} \times D^{m-k}$ of index $k$ attached, see [7, Theorem 17.5]. Note that this uses as data the embed$\operatorname{ding} S \rightarrow \partial M^{a}$ of a sphere $S:=S^{k-1}$ with trivial normal bundle $T_{S}^{\perp}\left(\partial M^{a}\right)$ and, see $[27$, p. 24], a bundle isomorphism

$$
\varphi: S \times \mathbb{R}^{m-k} \longrightarrow T_{S}^{\perp}\left(\partial M^{a}\right) .
$$

Then by excision [27, Section 2.3], one has

$$
H_{\bullet}\left(M^{b}, M^{a}\right) \cong H_{\bullet}\left(D^{k}, \partial D^{k}\right) .
$$

Here $H_{\bullet}(X, A) \equiv H_{\bullet}(X, A ; \mathbb{F})$ denotes the relative homology chain complex of a pair $(X, A)$. (Unless stated otherwise, the coefficients are in a field $\mathbb{F}$.) By subadditivity $\left[27\right.$, Lemma 2.14] for the long exact homological sequence of the pair $\left(M^{b}, M^{a}\right)$, one gets the inequality

$$
P\left(M^{a}\right)+P\left(M^{b}, M^{a}\right) \succeq P\left(M^{b}\right)
$$

of Poincaré polynomials, with relative Poincaré polynomial $P\left(M^{b}, M^{a}\right)(t)=t^{k}$. The symbol $\succeq$ means existence of a polynomial $Q$ with nonnegative coefficients such that

$$
\left(P\left(M^{a}\right)+P\left(M^{b}, M^{a}\right)-P\left(M^{b}\right)\right)(t)=(1+t) Q(t) .
$$

This implies, in particular, that $P\left(M^{a}\right) \neq P\left(M^{b}\right)$.

Hence, if $f$ is exhaustive (in particular, if $M$ is compact) and $M_{a}^{b}$ contains a critical level $f^{-1}(c)$ with one critical point, then $M^{a}$ is not homotopy equivalent to 
$M^{b}$. As we have seen above, for the level sets $\partial M^{a}=f^{-1}(a)$ this is no longer the case.

We note that if $M$ is non-compact, it may happen that the level and sublevel sets change their topology even in the absence of critical points. This phenomenon occurs, for instance, in the 3-body problem and in that case is due to the so-called critical points at infinity [2]. To avoid this situation, but to include a large class of functions on non-compact manifolds, we shall assume throughout the paper, without always mentioning this explicitly, that $f$ satisfies the following assumptions; $\mathrm{cf}$. $[28]$.

Assumptions 2.2. 1. There exists a Riemannian metric on $M$ such that $M$ is complete with respect to this metric and for every $S \subset M$ on which $|f|$ is bounded, but the norm $\|\operatorname{grad} f\|$ is not bounded away from zero, there is a critical point of $f$ in the closure of $S$.

2. The integer homology groups of each level set $\partial M^{b}=f^{-1}(b)$ are finitely generated.

Remark 2.3. The first assumption is known as the Palais-Smale condition [28]. Under this condition, the usual Morse theory applies, even if the level sets $f^{-1}(a)$ are not compact. The second condition is technical and is needed for dimension counting. It implies, for instance, that homology groups with coefficients in a field $\mathbb{F}$ are finite-dimensional; see Lemma 2.4. We note that when $f$ is a proper function, that is, when all the sets $M_{a}^{b}=f^{-1}[a, b]$ are compact (in particular, when $f$ is exhaustive), Assumptions 2.2 are satisfied. Non-compact examples can be found in Sections 3 and 4.

We will need the following lemma, where the above notation is understood.

Lemma 2.4. $\left({ }^{2}\right)$ Let $x_{c}$ be a non-degenerate critical point of $f$ with

$$
k=\operatorname{index}\left(f, x_{c}\right)
$$

and let $\mathbb{F}$ be a field. Assume that values a and $b$ are chosen such that $x_{c}$ is the only critical point in $M_{a}^{b}$. Then we have (under Assumptions 2.2)

$$
\operatorname{dim} H_{\ell}\left(\partial M^{b} ; \mathbb{F}\right)=\operatorname{dim} H_{\ell}\left(\partial M^{a} ; \mathbb{F}\right)+j_{\ell},
$$

where $j_{\ell}=0$ if $\ell \notin\{k-1, k, m-k-1, m-k\}$. Moreover,

1) if $2 k<m-1$, then either $j_{k-1}=0$ and $j_{k}=1$, or $j_{k-1}=-1$ and $j_{k}=0$;

2) if $2 k=m-1$, then $j_{k-1}=0$ and $j_{k}=1,2$, or $j_{k-1}=-1$ and $j_{k}=0,1$.

$\left({ }^{2}\right)$ Compare with [25, Lemma 2] and [31, Proposition 4.19]. 
Example 2.5. For instance, the data $j_{1}=1, j_{0}=0,2 k<m-1$ can be realised as a bifurcation from $S^{3}$ to $S^{2} \times S^{1}$. Other possibilities can be realised as bifurcations of the following form: $S^{3} \sqcup S^{3} \rightarrow S^{3}, S^{2} \sqcup S^{2} \rightarrow S^{2}, \mathbb{R}^{2} \sqcup \mathbb{R}^{2} \rightarrow S^{1} \times \mathbb{R}, S^{2} \rightarrow \mathbb{T}^{2}$, and $S^{2} \rightarrow K L$ (here $K L$ stands for the Klein bottle).

Proof. First we consider the case of an arbitrary index $k$. We will come back to the condition $2 k \leq m-1$ at the end of the proof.

From standard Morse theory, the space $M_{a}^{b}$ is homotopy equivalent to $\partial M^{a}$ with a $k$-cell $D^{k}$ attached. Analogously, we have that $M_{a}^{b}$ is homotopy equivalent to $\partial M^{b}$ with an $m-k$-cell $D^{m-k}$ attached. Hence,

$$
\begin{aligned}
& H_{\ell}\left(M_{a}^{b}, \partial M^{a}\right)=H_{\ell}\left(D^{k}, \partial D^{k}\right)=\mathbb{F}^{\delta_{k}(\ell)} \text { and } \\
& H_{\ell}\left(M_{a}^{b}, \partial M^{b}\right)=H_{\ell}\left(D^{m-k}, \partial D^{m-k}\right)=\mathbb{F}^{\delta_{m-k}(\ell)},
\end{aligned}
$$

where we use $\mathbb{F}$ as the coefficients for homology groups; $\delta_{k}(\ell)$ stands for the Kronecker delta. From the exact homology sequences of the pairs $\left(M_{a}^{b}, \partial M^{a}\right)$ and $\left(M_{a}^{b}, \partial M^{b}\right)$, we therefore get that the following sequences are exact:

$$
\begin{aligned}
& \mathbb{F}^{\delta_{k}(\ell+1)} \stackrel{\partial}{\longrightarrow} H_{\ell}\left(\partial M^{a}\right) \stackrel{i_{\ell}^{a}}{\longrightarrow} H_{\ell}\left(M_{a}^{b}\right) \stackrel{j_{\ell}^{a}}{\longrightarrow} \mathbb{F}^{\delta_{k}(\ell)}, \\
& \mathbb{F}^{\delta_{m-k}(\ell+1)} \stackrel{\partial}{\longrightarrow} H_{\ell}\left(\partial M^{b}\right) \stackrel{i_{\ell}^{b}}{\longrightarrow} H_{\ell}\left(M_{a}^{b}\right) \stackrel{j_{\ell}^{b}}{\longrightarrow} \mathbb{F}^{\delta_{m-k}(\ell)} .
\end{aligned}
$$

Let

$$
a_{\ell}:=\operatorname{dim}\left(H_{\ell}\left(\partial M^{a}\right)\right), b_{\ell}:=\operatorname{dim}\left(H_{\ell}\left(\partial M^{b}\right)\right), c_{\ell}:=\operatorname{dim}\left(H_{\ell}\left(M_{a}^{b}\right)\right) .
$$

It follows that $a_{\ell}=c_{\ell}=b_{\ell}$ if $\ell \notin\{k-1, k, m-k-1, m-k\}$. This proves the first statement. To prove the remaining statements, consider the homology classes $\left[\partial D^{k}\right] \in$ $H_{k-1}\left(\partial M^{a}\right)$ and $\left[\partial D^{m-k}\right] \in H_{m-k-1}\left(\partial M^{b}\right)$ under the boundary homomorphisms $\partial$. There are two possibilities for each of these classes: either they represent a trivial or non-trivial element in $H_{k-1}\left(\partial M^{a}\right)$, respectively, $H_{k-1}\left(\partial M^{b}\right)$. (We note that in the closed and orientable case, Poincaré's duality prohibits two of these four options, but we do not make these assumptions here; cf. Example 2.5 and Remark 2.6.)

Assume that $\partial\left[D^{k}\right]=0$ and $\partial\left[D^{m-k}\right]=0$. Then there exist $\alpha_{k}$ in $H_{k}\left(\partial M^{a}\right)$ such that $j_{k}^{a}\left(\alpha_{k}\right)=\left[D^{k}\right]$. In particular, $\alpha_{k}$ is not in the kernel of $j_{k}^{a}$ and, by exactness, not in the image of $i_{k}^{a}$. It follows that $a_{k}=c_{k}-1$. Similarly, $b_{m-k}=c_{m-k}-1$. In all other dimensions, $a_{\ell}=c_{\ell}=b_{\ell}$. Hence, $j_{k}=1, j_{m-k}=-1$ and $j_{\ell}=0$ for $\ell \neq k, m-k$.

Let $\partial\left[D^{k}\right] \neq 0$ and $\partial\left[D^{m-k}\right] \neq 0$. Then $a_{k-1}=c_{k-1}+1$ and $b_{m-k-1}=c_{m-k-1}+1$. In all other dimensions, $a_{\ell}=c_{\ell}=b_{\ell}$. It follows that $j_{k-1}=-1, j_{m-k-1}=1$ and $j_{\ell}=0$ for $\ell \neq k-1, m-k-1$.

Now let $\partial\left[D^{k}\right]=0$ and $\partial\left[D^{m-k}\right] \neq 0$. Then $a_{k}=c_{k}-1$ and $b_{m-k-1}=c_{m-k-1}+1$. In all other dimensions, $a_{\ell}=c_{\ell}=b_{\ell}$. Now if $k \neq m-k-1$, then $j_{k}=j_{m-k-1}=1$ and 
$j_{\ell}=0$ for $\ell \neq k, m-k-1$. If $k=m-k-1$, that is, if $2 k=m-1$, then $j_{k}=2$ and $j_{\ell}=0$ for $\ell \neq k$.

In the remaining case of $\partial\left[D^{k}\right] \neq 0$ and $\partial\left[D^{m-k}\right]=0$, we have the following: $a_{k-1}=c_{k-1}+1$ and $b_{m-k}=c_{m-k}-1$. If $k-1 \neq m-k$, then $j_{k-1}=j_{m-k}=-1$ and $j_{\ell}=0$ for $\ell \neq k-1, m-k$. If $k-1=m-k$, that is, if $2 k=m+1$, then $j_{m-k}=-2$ and $j_{\ell}=0$ for $\ell \neq m-k$.

Collecting the above results and assuming $2 k<m-1$, respectively, $2 k=m-1$, yields the remaining statements of the lemma.

Remark 2.6. If $M$ is closed and orientable, then the change in homology groups happens according to Poincaré duality, and we can specify Lemma 2.4 further as follows. (As above, $k$ stands for the index of the critical point and $m$ is the dimension of $M$.)

- If $2 k \neq m, m-1, m+1$, then either $j_{k-1}=j_{m-k}=-1$ and the remaining $j_{\ell}=0$, or $j_{k}=j_{m-k-1}=1$ and $j_{\ell}=0$ for all $\ell \neq k, m-k-1$.

- If $2 k=m-1$, then $j_{k}=2$ and the remaining $j_{\ell}=0$, or $j_{k-1}=j_{m-k}=-1$ and $j_{\ell}=0$ for $\ell \neq k-1, m-k$.

- Similarly, if $2 k=m+1$, then $j_{m-k}=-2$ and $j_{\ell}=0$ for all $\ell \neq k$, or $j_{k}=j_{m-k-1}=$ 1 and the remaining $j_{\ell}=0$.

Remark 2.7. In the case of the middle dimension, we can similarly obtain the following statement. Assume that $M_{a}^{b}$ contains a single critical point of the index $k=m / 2$. Then the levels $\partial M^{b}$ and $\partial M^{a}$ have different homology groups if exactly one of the classes $\left[\partial D^{k}\right] \in H_{k-1}\left(\partial M^{a}\right)$ and $\left[\partial D^{m-k}\right] \in H_{m-k-1}\left(\partial M^{b}\right)$ vanishes. (Here the above notation and Assumptions 2.2 are understood.)

Observe that the function $g=-f$ has the same level sets as the function $f$; moreover, each index- $k$ critical point of $f$ is also a critical point of $g$ of index $m-k$. From Lemma 2.4, we get the following result.

Theorem 2.8. Let $f$ be a Morse function on an $m$-manifold $M$ with sublevel sets $M^{b}=f^{-1}(-\infty, b]$ and $M_{a}^{b}=f^{-1}[a, b]$. Assume that $f$ satisfies Assumptions 2.2 and that the set $M_{a}^{b}$ contains $N \geq 1$ critical points of the same index $k$ and no critical points of some other index. If the homotopy type of $\partial M^{b}$ and of $\partial M^{a}$ coincide, then $k=m / 2$ (in particular, $m$ is even).

More generally, we have the following theorem.

Theorem 2.9. Let $f$ be a Morse function on an $m$-manifold $M$. Assume that the set $M_{a}^{b}$ contains a critical point of index $k \neq m / 2$ such that there exists no other critical point in $M_{a}^{b}$ of index $k-1, k+1$ or $m-k$. Then, under Assumptions 2.2 , the homotopy types of $\partial M^{b}$ and of $\partial M^{a}$ do not coincide. 
Proof. Let $x_{k}$ be a critical point of index $k$ in $M_{a}^{b}$. We shall assume that $k<m / 2$; otherwise, we consider the function $g=-f$, which has the same level sets as $f$. Let $x_{k^{\prime}}$ be any other critical point in $M_{a}^{b}$, if it exists.

Let $j_{\ell}\left(x_{k}\right)$ denote the change that occurs in the $\ell$-th Betti numbers when $f$ passes $x_{k}$. Then, according to Lemma 2.4, either $j_{k-1}\left(x_{k}\right)=-1$ or $j_{k}\left(x_{k}\right)=1,2$ (these possibilities are not mutually exclusive). Moreover, we always have $j_{k-1}\left(x_{k}\right) \leq 0$ and $j_{k}\left(x_{k}\right) \geq 0$.

For the critical point $x_{k^{\prime}}$ of index $k^{\prime}$, we may observe a change in the Betti numbers only in the following dimensions: $\left\{k^{\prime}-1, k^{\prime}, m-k^{\prime}-1, m-k^{\prime}\right\}$. Thus, if $k-1$ and $k$ do not belong to $\left\{k^{\prime}-1, k^{\prime}, m-k^{\prime}-1, m-k^{\prime}\right\}$, the statement follows. Since $k^{\prime} \neq k+1, k-1, m-k$, the remaining possibilities are $k^{\prime}=k, k^{\prime}=m-k-1$, and $k^{\prime}=m-k+1$. We observe that

- If $k^{\prime}=k$, then by Lemma $2.4, j_{k-1}\left(x_{k^{\prime}}\right) \leq 0$ and $j_{k}\left(x_{k^{\prime}}\right) \geq 0$.

- If $k^{\prime}=m-k+1$, then $m-k^{\prime}=k-1$. Applying Lemma 2.4 to the function $g=-f$, we get that $j_{k-1}\left(x_{k^{\prime}}\right) \leq 0$ and $j_{k}\left(x_{k^{\prime}}\right)=0$.

- Finally, consider the case $k^{\prime}=m-k-1$. If $k^{\prime}<m / 2$, then $k^{\prime}=k$. This case was considered earlier. If $k^{\prime}>m / 2$, then $m-k^{\prime}=k+1<m / 2$. Moreover, $j_{k}\left(x_{k^{\prime}}\right) \geq 0$ and $j_{k-1}\left(x_{k^{\prime}}\right)=0$. The remaining case $k^{\prime}=m / 2$ is not possible since then $k+1=m / 2=k^{\prime}$.

We conclude that in all these three cases $j_{k-1}\left(x_{k^{\prime}}\right) \leq 0$ and $j_{k}\left(x_{k^{\prime}}\right) \geq 0$, and the initial change that occurs in the Betti numbers when passing $x_{k}$ cannot be compensated. The result follows.

We note that the critical points in Theorems 2.8 and 2.9 may occur at different level sets.

\section{Energy levels in classical mechanics}

\subsection{Mechanical systems on vector bundles}

Consider a rank $n$ vector bundle

$$
\pi: E \longrightarrow N
$$

over a connected $n$-manifold $N$ without boundary. The manifold $N$ and the bundle $\pi: E \rightarrow N$ are assumed to be orientable.

We will be interested in the topology change of level sets of a 'Hamiltonian' function on $E$ of the following form (3.2) (which includes the class of natural mechanical systems and natural mechanical systems with magnetic terms)

$$
H=K+V \circ \pi
$$


where $K$ is a Riemannian bundle metric on $E$ and $V$ is a Morse function on $N$. We shall assume that both $H$ and $V$ satisfy Assumptions 2.2. We will need the following result, which specifies how the homology groups of the level sets $H^{-1}(h)$ change when passing an index- $n$ critical point.

Lemma 3.1. Let $x_{c}$ be a non-degenerate local maximum of $V$ such that there are no other critical points on $V^{-1}\left(h_{c}\right), h_{c}=V\left(x_{c}\right)=H\left(x_{c}, 0\right)$. Let $\mathbb{F}$ be a coefficient field and $\varepsilon>0$ be sufficiently small. Then the $(n-1)$-Betti number changes according to

$$
b_{n-1}\left(H^{-1}\left(h_{c}+\varepsilon\right) ; \mathbb{F}\right)=b_{n-1}\left(H^{-1}\left(h_{c}-\varepsilon\right) ; \mathbb{F}\right)+j_{n-1},
$$

where $j_{n-1}=-1$ if $x_{c}$ is not a global maximum and $j_{n-1} \in\{-1,0,1\}$ if $x_{c}$ is a global maximum.

Proof. Let $x_{c}$ be a local maximum of $V$ and $h_{c}$ be the corresponding critical value. By Assumptions 2.2, for all $\varepsilon>0$ small, $h_{c}$ is the only critical value of $V$ (and therefore also of $H)$ in $\left[h_{c}-\varepsilon, h_{c}+\varepsilon\right]$. We observe that:

1. By applying the Morse Lemma [27, Theorem 1.12] to $V$, there is a closed neighborhood $U \subseteq N$ of $x_{c}$ and a suitable chart $\psi: U \rightarrow \psi(U)=D^{m} \subseteq \mathbb{R}^{m}$ with $\psi\left(x_{c}\right)=$ 0 , such that $V \circ \psi^{-1}(x)=h_{c}-\|x\|^{2}$.

2. Following a proof of the Morse lemma (for the function $H$ ), we can find a closed neighborhood $\widetilde{U}$ of $\tilde{x}_{c}:=\left(x_{c}, 0\right)$ and a local trivialization $\varphi: \widetilde{U} \rightarrow D^{n} \times D^{n}$ of (3.1) such that $\psi \circ \pi=\pi_{1} \circ \varphi$ for $\pi_{1}(x, y):=x$ and

$$
H \circ \varphi^{-1}(x, y)=h_{c}+\|y\|^{2}-\|x\|^{2} \quad\left((x, y) \in D^{n} \times D^{n}\right) .
$$

If $\varepsilon>0$ is small, then the intersections with $W=\pi^{-1}(U)$ of the levels

$$
\Sigma_{ \pm}:=H^{-1}\left(h_{c} \pm \varepsilon\right)
$$

are contained in $\widetilde{U}$; moreover, they are the images of diffeomorphisms

$$
\hat{f}_{-}: S^{n-1} \times D^{n} \longrightarrow \Sigma_{-} \cap W, \text { respectively, } \hat{f}_{+}: D^{n} \times S^{n-1} \longrightarrow \Sigma_{+} \cap W
$$

see Figure 3.1.

3. The $(2 n-1)$-manifolds with boundary

$$
T_{ \pm}:=\Sigma_{ \pm} \backslash \operatorname{int}(W)
$$

are naturally diffeomorphic. 


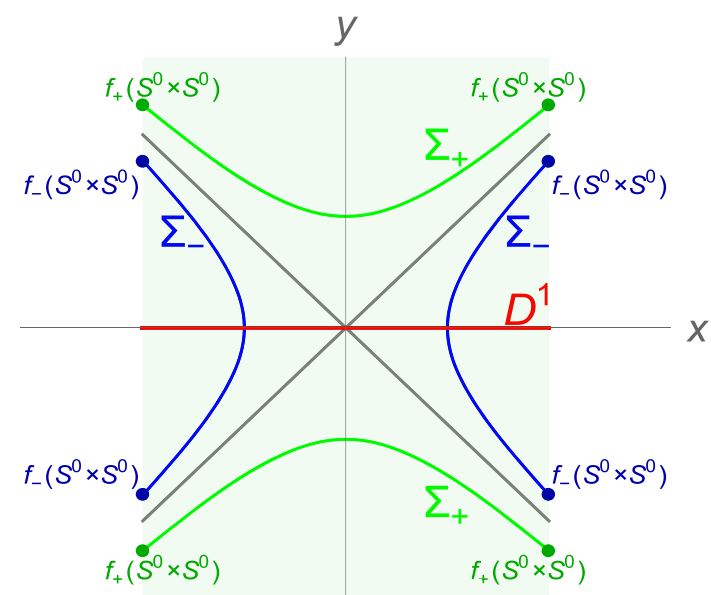

Figure 3.1. The neighborhood $\widetilde{U} \subset \pi^{-1}(U)$ of the critical point $\widetilde{x}_{c}$ for $n=\operatorname{dim}(N)=1$.

The third statement follows, for instance, from the following construction. Specifically, consider a regular level set $H^{-1}(h)$. It projects to the corresponding Hill region

$$
B_{h}:=\{x \in N \mid V(x) \leq h\},
$$

which is a manifold with boundary. The level set $H^{-1}(h)$ can be viewed as a sphere bundle with fibers $S_{r(x)}^{n-1}$ over $x \in B_{h}$ of radius $r(x)=\sqrt{2(h-V(x))}$, thus collapsed over $\partial B_{h}$.

By 1) and 2), using the chart $(\widetilde{U}, \varphi)$, we can write

$$
\Sigma_{-} \cong T_{-} \cup_{f_{-}} S^{n-1} \times D^{n} \text { and } \Sigma_{+} \cong T_{+} \cup_{f_{+}} D^{n} \times S^{n-1}
$$

where the attaching maps for the respective boundary components are the diffeomorphisms

$$
f_{ \pm}:=\left.\hat{f}_{ \pm}\right|_{S^{n-1} \times S^{n-1}}: S^{n-1} \times S^{n-1} \longrightarrow \partial T_{ \pm}, \quad(x, y) \mapsto(x, \sqrt{1 \pm \varepsilon} y) .
$$

From this description it follows that under the map $f_{-}$, the boundary of the $n$-disk $\{x\} \times D^{n}$ is mapped to a fiber of the collapsed sphere bundle $\pi: T_{-} \rightarrow \pi\left(T_{-}\right)$. On the other hand, $f_{+}\left(\partial D^{n} \times\{y\}\right)$ is a cross section. We note that

$\left[\{x\} \times D^{n}\right]$ generates $H_{n}\left(S^{n-1} \times D^{n}, S^{n-1} \times S^{n-1} ; \mathbb{F}\right)$, if $x \in S^{n-1}$;

$\left[D^{n} \times\{y\}\right]$ generates $H_{n}\left(D^{n} \times S^{n-1}, S^{n-1} \times S^{n-1} ; \mathbb{F}\right)$, if $y \in S^{n-1}$.

Consider the homology exact sequences of the pairs $\left(\Sigma_{ \pm}, T_{ \pm}\right)$:

$$
\mathbb{F} \stackrel{\partial_{*}}{\longrightarrow} H_{n-1}\left(T_{ \pm} ; \mathbb{F}\right) \longrightarrow H_{n-1}\left(\Sigma_{ \pm} ; \mathbb{F}\right) \longrightarrow 0 .
$$


Below we shall use this sequence, together with the information about the attaching maps $f_{ \pm}$and some additional properties to compare the $(n-1)$-th homology groups of $\Sigma_{+}$and $\Sigma_{-}$.

First, consider the case of a global maximum. In this case, the statement follows from the exactness of (3.4). Indeed, the exactness of this sequence implies that $b_{n-1}\left(T_{ \pm} ; \mathbb{F}\right)=b_{n-1}\left(\Sigma_{ \pm} ; \mathbb{F}\right)$ or $b_{n-1}\left(T_{ \pm} ; \mathbb{F}\right)=b_{n-1}\left(\Sigma_{ \pm} ; \mathbb{F}\right)+1$. (Recall that, by the assumption, the homology groups of $T_{ \pm}$and $\Sigma_{ \pm}$are finite dimensional $\mathbb{F}$-vector spaces.) But by Observation 3) above, the spaces $T_{-}$and $T_{+}$are diffeomorphic and, in particular, $b_{n-1}\left(T_{+} ; \mathbb{F}\right)=b_{n-1}\left(T_{-} ; \mathbb{F}\right)$. It follows that $b_{n-1}\left(\Sigma_{+} ; \mathbb{F}\right)=b_{n-1}\left(\Sigma_{-} ; \mathbb{F}\right)+$ $j_{n-1}$, where $j_{n-1} \in\{-1,0,1\}$.

Now consider the case when $x_{c}$ is a local, but not a global maximum. We observe that the following properties hold:

A) Let $B_{ \pm}:=B_{h_{c} \pm \varepsilon}$. The intersection $\partial \pi\left(T_{+}\right) \cap \partial B_{+} \neq \varnothing$.

B) For any $x \in \pi\left(T_{-}\right)$, there is a homotopy within $T_{-}$between the fiber $S_{r(x)}^{n-1}$ c $\rightarrow T_{-}$over $x$ (with $\left.r=\sqrt{2\left(h_{c}-\varepsilon-V(x)\right)}\right)$ and a point.

To prove A), we note that $h_{c}<h_{c}+\varepsilon<\sup _{q} V(q)$, so that $\partial B_{+} \neq \varnothing$, and that $\pi\left(T_{+}\right)=B_{+} \backslash U$.

To prove B), we construct a homotopy $p: S^{n-1} \times[0,1] \rightarrow T_{-}$which projects to a path $\pi \circ p:[0,1] \rightarrow B_{-}$from $x_{c}$ to a point of $\partial B_{-} \backslash \psi^{-1}\left(S_{\sqrt{\varepsilon}}^{n-1}\right)$.

We note that properties $\mathrm{A}$ ) and $\mathrm{B}$ ) do not hold if $x_{c}$ is a global maximum. If $x_{c}$ is only a local maximum, then $b_{n-1}\left(\Sigma_{+} ; \mathbb{F}\right)=b_{n-1}\left(\Sigma_{-} ; \mathbb{F}\right)-1$, as we now show.

- Consider the case of $\Sigma_{+}$. The first of the maps in (3.4) is given by a boundary homomorphism $\partial_{*}$ on $H_{n}\left(D^{n} \times S^{n-1}, S^{n-1} \times S^{n-1} ; \mathbb{F}\right) \cong \mathbb{F}$. We claim that the image of $\partial_{*}$ is non-trivial in this case. Indeed, by $\left.\mathrm{A}\right),\left[D^{n} \times\{y\}\right] \in H_{n}\left(D^{n} \times\right.$ $\left.S^{n-1}, S^{n-1} \times S^{n-1} ; \mathbb{F}\right) \backslash\{0\}$. We observed above that $f_{+}\left(\partial D^{n} \times\{y\}\right)$ is a cross section over $\pi\left(f_{+}\left(\partial D^{n} \times\{y\}\right)\right)$, where $\pi$ is defined in (3.1). It follows that

$$
\pi_{*}\left(\partial_{*}\left[f_{+}\left(D^{n} \times\{y\}\right)\right]\right)=[\partial U] .
$$

In particular, we have that $\partial_{*}\left[f_{+}\left(D^{n} \times\{y\}\right)\right] \in H_{n-1}\left(T_{+}\right)$is non-zero, using B). From the exactness of (3.4) it follows that the map $H_{n-1}\left(T_{+}\right) \rightarrow H_{n-1}\left(\Sigma_{+}\right)$is not injective. However, it is surjective by virtue of the last arrow in (3.4).

- Consider the remaining case of $\Sigma_{-}$. We observe that the map

$$
H_{n-1}\left(T_{-}\right) \longrightarrow H_{n-1}\left(\Sigma_{-}\right)
$$

is bijective; indeed, from $\mathrm{C}$ ) it follows that image $\left(\partial_{*}\right)=0$ in this case.

Recall that the homology groups of $T_{ \pm}, \Sigma_{ \pm}$are finite dimensional $\mathbb{F}$-vector spaces and that $T_{-}$and $T_{+}$are diffeomorphic. By counting dimensions, we conclude that $b_{n-1}\left(\Sigma_{+} ; \mathbb{F}\right)=b_{n-1}\left(\Sigma_{-} ; \mathbb{F}\right)-1$. 
Combining Theorem 2.8 and Lemma 3.1, we get

Corollary 3.2. Consider a function on $E$ of the form

$$
H=K+V \circ \pi
$$

where $K$ is a Riemannian bundle metric on $E$. Let $x_{c}$ be a non-degenerate critical point of $V$ that is not a global maximum. Assume that $x_{c}$ is the only critical point on $V^{-1}\left(h_{c}\right)$, for $h_{c}=V\left(x_{c}\right)=H\left(x_{c}, 0\right)$. Then the topology of $H^{-1}(h)$ changes when $h$ passes the critical value $h_{c}$.

Remark 3.3. Assume that the base manifold $N$ is compact and 2-dimensional. Then for $h<\max V$, the corresponding energy levels $H^{-1}(h)$ are topologically classified by the Euler characteristic of the Hill region $B_{h}$; see [3]. This gives another proof of Corollary 3.2 in this case, since the Euler characteristic of $B_{h}$ changes when passing a Morse critical point.

Corollary 3.4. Consider a function on $E$ of the form

$$
H=K+V \circ \pi
$$

where $K$ is a Riemannian bundle metric on $E$. Let $x_{1}, \ldots, x_{L} \in V^{-1}\left(h_{c}\right)$ be the nondegenerate global maxima of the function $V$ on $N$. If $L \geq 3$, then the topology of $H^{-1}(h)$ changes when $h$ passes the critical value $h_{c}$.

Remark 3.5. We note that Lemma 3.1 and Corollaries 3.2, 3.4 hold even when the bundle (3.1) is not orientable (but the base $N$ still is).

Below we shall study in more detail the case of a global maximum of $V$. In addition to the orientability of the manifold $N$ and the bundle $\pi: E \rightarrow N$, we shall assume that the base manifold $N$ is compact. Under these assumptions, the Euler number $e(E)$ of $\pi: E \rightarrow N$ is defined.

The following result specifies when the topology of $H^{-1}(h)$ changes when passing a global maximum of $V$ or a number $N$ of global maxima, which are on the same energy level.

Theorem 3.6. Let $N$ be a closed orientable $n$-manifold and $\pi: E \rightarrow N$ be an orientable rank $n$ vector bundle over $N$. Consider the function $H=K+V \circ \pi: E \rightarrow \mathbb{R}$ on $E$ and let $x_{1}, \ldots, x_{L} \in V^{-1}\left(h_{c}\right)$ be the non-degenerate global maxima of the function $V$ on $N$. Then the topology of $H$ changes when $H$ passes the critical value $h_{c}$ if one of the following conditions is satisfied

1) $L=1$ and the Euler number $e(E)$ is not equal to \pm 1 ;

2) $L=2$ and the Euler number $e(E)$ does not vanish;

3) $L>2$. 
Proof. The case $L>2$ was considered earlier. The proof in the other cases relies on the homology exact sequence of a pair. In the case $L=1$, a special choice of a coefficient group is made; specifically $-\mathbb{Z}_{k}$, where $k=e(E)$ is the Euler number. Details are given in Appendix A; see Theorem A.5.

Corollary 3.7. Let $N$ be a closed orientable $n$-manifold and $\pi: E \rightarrow N$ be an orientable rank $n$ vector bundle over $N$. If the Euler number $e(E) \neq \pm 1$, then the topology of $H=K+V \circ \pi: E \rightarrow \mathbb{R}$ changes whenever $H$ passes a critical level $H^{-1}\left(h_{c}\right)$ with one (and only one) non-degenerate critical point.

For cotangent bundles, we have the following result.

Corollary 3.8. If $E=T^{*} N$ is the cotangent bundle of a closed orientable manifold $N$ and the Euler characteristic $\chi(N) \neq \pm 1$, then the topology of the level sets for $H=K+V \circ \pi: T^{*} N \rightarrow \mathbb{R}$ always changes when $H$ passes a simple critical level. This is the case, in particular, if

(1) the dimension $\operatorname{dim}(N)$ is odd or $\operatorname{dim}(N)=2 k$, where $k$ is odd;

(2) the Betti number $b_{\operatorname{dim}(N) / 2}(N)$ is even.

Remark 3.9 .

(1) We remark that Theorem 3.6 and Corollary 3.7 do not hold if one does not make any assumptions on $N$ or $E$. For instance, consider the tautological line bundle over $N=\mathbb{C P}^{1}$. Then, for any smooth function $V$ on $\mathbb{C P}^{1}$ (with a unique non-degenerate maximum), we have that

$$
H^{-1}\left(h_{\max }+\varepsilon\right) \cong H^{-1}\left(h_{\max }-\varepsilon\right) \cong S^{3},
$$

since $\pi: H^{-1}\left(h_{\max }+\varepsilon\right) \rightarrow \mathbb{C P}^{1}$ is isomorphic to the Hopf bundle.

(2) By considering rank 4 vector bundles $\pi: E \rightarrow S^{4}$, one can even have a situation when $H^{-1}\left(h_{\max }+\varepsilon\right)$ is homeomorphic to $H^{-1}\left(h_{\max }-\varepsilon\right) \cong S^{7}$, but not diffeomorphic to it; this follows from [26].

(3) For cotangent bundles, the situation is a bit different.

First, we note that Corollary 3.8 applies to all 2-dimensional orientable surfaces (in this case, the Euler characteristic of $N$ is even), parallelizable manifolds $N$ (in particular, to all Lie groups), and odd-dimensional manifolds.

Thus, there are no counterexamples in dimensions $n=3$ or less.

We conjecture that the 4 -manifold $\mathbb{C P}^{2} \# \mathbb{T}^{4}$ with Euler characteristic

$$
\chi\left(\mathbb{C P}^{2} \# \mathbb{T}^{4}\right)=\chi\left(\mathbb{C P}^{2}\right)+\chi\left(\mathbb{T}^{4}\right)-\chi\left(S^{4}\right)=3-2=1
$$

is a counterexample.

We note that in the non-orientable case $N=\mathbb{R} P^{2}, \chi\left(\mathbb{R} P^{2}\right)=1$, but $H^{-1}\left(h_{\max }-\right.$ $\varepsilon)$ and $H^{-1}\left(h_{\max }+\varepsilon\right)$ are not diffeomorphic; they are diffeomorphic to $S^{2} \times S^{1}$ and the lens space $L(4,1)$, respectively [18]. 
In fact, for a class of bundles, including bundles over spheres, we have a much stronger statement, which follows from Adams' result [1].

Proposition 3.10. Consider an orientable rank $n$ vector bundle $\pi: E \rightarrow N$ over a closed orientable manifold $N$ and a Morse function on $E$ of the form

$$
H=K+V \circ \pi
$$

If the restriction $\pi: \pi^{-1}(N \backslash U) \rightarrow N \backslash U$, where $U$ is a small disk in $N$, is a trivial bundle and $n \neq 2,4$ or 8 , then the topology of $H$ level sets changes when passing a simple critical level.

Proof. The statement follows from Adams' result ( $S^{n-1}$ is an $H$-space only in dimensions $n=1,2,4$ and 8) [1].

Corollary 3.11. If $N$ is a homotopy $n$-sphere, a situation of no topology change is possible only when $n=2,4$ or 8 .

\section{Applications}

\subsection{Quadratic spherical pendulum}

Let $S^{2}$ denote the unit sphere in $\mathbb{R}^{3}(x, y, z)$. Consider the Hamiltonian system on $T^{*} S^{2}$ given by the energy function

$$
H=\frac{1}{2}\langle p, p\rangle+V(z)
$$

where $V=z^{2}-z / 2$ is the potential. This Hamiltonian system is integrable and is called a quadratic spherical pendulum; it naturally appears in the context of integrable Hamiltonian systems with non-trivial monodromy [9]; see also [4] for the necessary background. The corresponding bifurcation diagram, that is, the set of the critical values of the energy-momentum map $(H, J): T^{*} S^{2} \rightarrow \mathbb{R}^{2}$, is depicted in Figure 4.1 ; here $J$ is the angular momentum about the $z$-axis — the first integral of the system.

The Hamiltonian function $H$ has two non-degenerate critical points, which correspond to the two maxima of the potential $\left.V\right|_{S^{2}}$. These points give rise to two critical level sets. From Theorem 3.6, we conclude that the topology of $H^{-1}(h)$ changes when passing each of these critical levels. The same is true when we compare the topology of $H^{-1}(h)$ below and above these two critical levels. Indeed, the Euler characteristic $\chi\left(S^{2}\right)=2$ is different from 0 and \pm 1 . In fact, it can be shown that these level sets are diffeomorphic to $S^{2} \times S^{1}, S^{3}$, and $\mathbb{R} P^{3}$. We note that a similar 


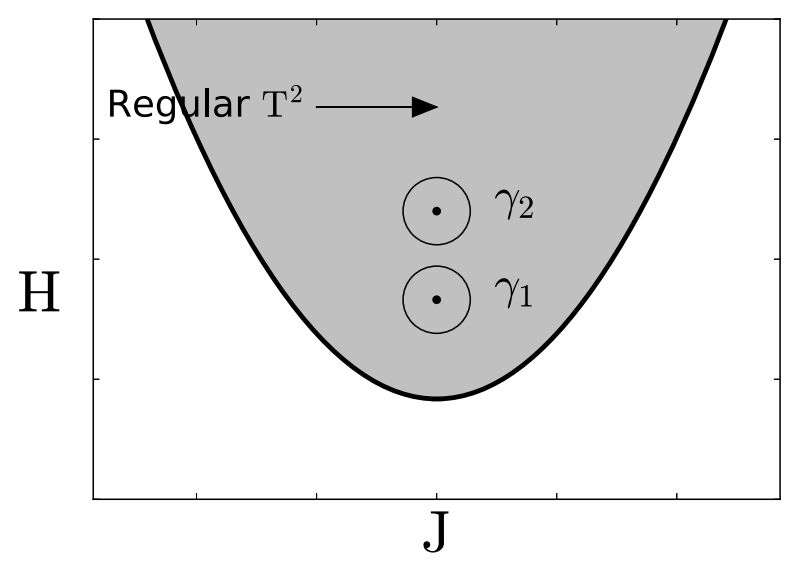

Figure 4.1. Bifurcation diagram of the energy-momentum map $(H, J)$, and the two curves $\gamma_{i}$ corresponding to non-trivial monodromy.

result applies also to the usual spherical pendulum, that is, when the potential $V(z)=z$.

One important consequence of the topology change for such system is the nontriviality of monodromy around the corresponding singular points; see [8] and [19] for more details and the background.

\subsection{Restricted three-body problem}

The planar circular restricted 3-body problem with mass ratio $\mu \in(0,1)$ can be written as an autonomous Hamiltonian system on $\mathbb{R}^{2} \times\left(\mathbb{R}^{2} \backslash\{(-\mu, 0),(0,1-\mu)\}\right)$ in a co-rotating reference frame; the Hamiltonian function is given by

$$
H=\frac{x^{2}+y^{\prime 2}}{2}-\frac{1}{2}\left(x^{2}+y^{2}\right)-\left(\frac{1-\mu}{r_{1}}+\frac{\mu}{r_{2}}\right),
$$

$r_{i}$ denoting the distances to the respective centers. There are five equilibrium points $L_{1}, \ldots, L_{5}$. These are the critical points of the potential function

$$
V=-\frac{1}{2}\left(x^{2}+y^{2}\right)-\left(\frac{1-\mu}{r_{1}}+\frac{\mu}{r_{2}}\right) .
$$

Each of these critical points gives rise to a bifurcation value for the energy function $H$. It is known that each such value gives rise to a topology change of the energy levels $H^{-1}(h)$, and it is not difficult to determine the homotopy types of these energy levels; the corresponding Hill regions are shown in Figure 4.2. Below we show how this result of the topology change follows from the theory developed in this paper. 


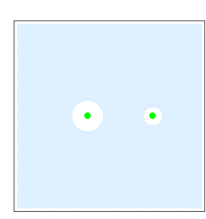

$S^{1} v S^{1}$

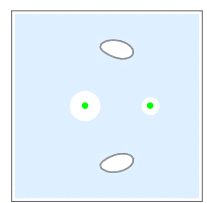

$S^{1} \vee S^{1} \vee S^{1} v S^{1}$

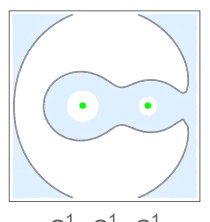

$S^{1} \vee S^{1} \vee S^{1}$

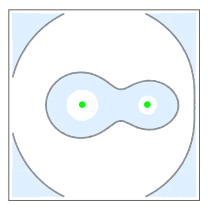

$S^{1} v S^{1} u S^{1}$

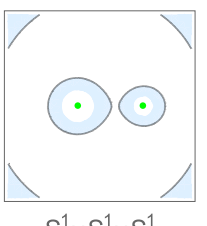

$S^{1} u S^{1} u S^{1}$

Figure 4.2. The Hill regions and their homotopy types.

First, we note that $L_{1}, L_{2}$ and $L_{3}$ are index 1 critical points for $V$ and hence also for $H$. Hence the topology changes when passing these critical values by Theorem 2.8. The critical points $L_{4}$ and $L_{5}$ are of index 2 , which is half the dimension of the phase space, and are on the same energy level; these points are related by the $\mathbb{Z}_{2}=\mathrm{O}(2) / \mathrm{SO}(2)$ symmetry of the problem. Since the base manifold is not compact and there are two critical points, the result follows.

\subsection{Planar $n$-body problem}

Consider the Newtonian $n$-body problem in $\mathbb{R}^{2}$. The Hamiltonian of this problem is given by the function

$$
H=\sum_{i=1}^{n} \frac{\left\|p_{i}\right\|^{2}}{2 m_{i}}-\sum_{i<j} \frac{G m_{i} m_{j}}{\left\|q_{j}-q_{i}\right\|}
$$

where $G$ is the gravitational constant. Reducing by the translational symmetry, we get a Hamiltonian system on $T^{*} Q$, where $Q=\mathbb{R}^{2 n-2} \backslash \triangle$ with $\triangle$ denoting the (reduced) collision set, that is, the set of points where $q_{i}=q_{j}$ for some $i \neq j$, reduced by translations.

Fixing a non-zero value of the angular momentum $L=\sum_{i} q_{i}^{x} p_{i}^{y}-q_{i}^{y} p_{i}^{x}$ and taking the quotient with respect to the $\mathrm{SO}(2)$ symmetry group, one gets the reduced symplectic manifold $M$ of dimension $4 n-6$. The Hamiltonian $H$ restricts to this manifold as a smooth function. Following Smale [32] and [33], we are interested in the topology of the level sets of $H$ on this reduced manifold. Specifically, we would like to answer the general question of whether the topology of $\left.H^{-1}(h)\right|_{M}$ always changes when passing a bifurcation level.

We observe that the manifold $M$ can be viewed as a vector bundle over $\mathbb{R}_{+} \times$ $\mathbb{C P}(n-2)$. Here $\mathbb{R}_{+}=(0, \infty)$ and each point of $\mathbb{R}_{+}$corresponds to fixing the moment of inertia

$$
I=\frac{1}{2} \sum_{i} m_{i}\left\|q_{i}\right\|^{2} .
$$


Using [2], the reduction of the Hamiltonian to $M$ can be rewritten in the following form:

$$
H=K+c^{2} /\left(4 \rho^{2}\right)-U(q) / \rho,
$$

where $K$ is a bundle metric, $c \neq 0$ is the value of $L, I=\rho$ and $U$ denotes the reduction of the potential to the projective space $I^{-1}(1) / \mathbb{S}^{1}$. From this description, it follows that the index $\lambda$ of each non-degenerate critical point is at most $2 n-4<\frac{1}{2}(4 n-6)=$ $2 n-3$. From Theorem 2.8, we get the following result, which also follows from McCord [22, Proposition 5.2].

Theorem 4.1. Consider the Hamiltonian $H$ on the reduced manifold $M$. Assume that $V$ defines a Morse function on $I^{-1}(1) / \mathbb{S}^{1}=\mathbb{C P}(n-2) \backslash \tilde{\Delta}$, so that $H$ is a Morse function as well. Assume, moreover, that a given critical level set $H^{-1}\left(h_{c}\right)$ contains a single critical point or two critical points of the same index (related by the $\mathbb{Z}_{2}=\mathrm{O}(2) / \mathrm{SO}(2)$ symmetry $)$. Then the topology of $H^{-1}(h)$ changes when passing this critical level.

Acknowledgements. We are very grateful to A. Albouy for useful discussions and his remarks, which led to an improvement of the original version of our article. The authors would also like to thank the anonymous referees and participants of A.T. Fomenko's seminar Modern Geometry Methods for useful comments and suggestions.

\section{A. Appendix}

The goal of this section is to prove Theorem 3.6 formulated in Section 3. Recall that we consider a smooth function of the form

$$
H=K+V \circ \pi
$$

on a rank $n$ vector bundle $\pi: E \rightarrow N$. Here $N$ is a connected $n$-manifold without boundary, $K$ is a Riemannian bundle metric on $E$ and $V$ is a Morse function on $N$. The manifold $N$ and the bundle $\pi: E \rightarrow N$ are assumed to be orientable. In this section, the manifold $N$ is assumed to be compact.

Observe that a section $s: N \rightarrow E$ in general position has finitely many zeros and that, by homogeneity, they can be assumed to be arbitrary close to some point $x \in N$. This shows that $E$ admits an almost global section, which is defined and non-zero everywhere on $N \backslash D$ (and also on $N \backslash\{x\}$ ), where $D$ is an arbitrary small disk containing $x$. 
We observe that for the unit sphere bundle $\tilde{\pi}=\left.\pi\right|_{S_{1} N}$, the degree of the map

$$
r \circ s: \partial D \cong S^{n-1} \longrightarrow S^{n-1} \cong \tilde{\pi}^{-1}(x)
$$

where $r$ is a retraction of $\tilde{\pi}^{-1}(D)$ onto the central fiber $\tilde{\pi}^{-1}(x)$, is equal to the intersection number of $s(N)$ and the zero section $N \subset E$.

We will need the following lemma.

Lemma A.1. ([11, Section 19.6B] and [14, Section 4.D]) The Euler number $e(E)$, that is, the pairing of the Euler class of $E$ with $N$, is given by the intersection number of the zero section $N \subset E$ and a section in general position. If $E=T^{*} N$ is the cotangent bundle, the Euler number equals the Euler characteristic of $N$.

We will also need the following result.

Lemma A.2. Let $\pi: E \rightarrow B$ be an orientable $n$-vector bundle over a manifold $B$ (possibly with boundary). Assume that there exists a global, everywhere non-zero section $s$ of $\pi$. Let $\tilde{\pi}=\left.\pi\right|_{S_{1} B}: S_{1} B \rightarrow B$ be the unit sphere bundle of $\pi$ (with respect to some bundle metric). Then, for any coefficient group $G$, the relative homology groups of $\left(S_{1} B, \partial S_{1} B\right)$ are the same as for the direct product $\left(B \times S^{n-1}, \partial B \times S^{n-1}\right)$. Moreover, for all $b \in B$ and any $G, \tilde{\pi}^{-1}(b)$ represents a non-trivial homology class in $H_{n-1}\left(S_{1} B ; G\right)$.

Proof. Take any simplicial decomposition $K$ of $B$ and the standard cellular decomposition $\{p t\} \cup D^{n-1}$ of $S^{n-1}$. Then construct a cellular decomposition of $S_{1} B$ as follows. For any simplex $c_{k} \in K$, consider the preimage $\pi^{-1}\left(c_{k}\right)$. It is a direct product $c_{k} \times \mathbb{R}^{n}$. Without loss of generality, the section $s$ has the form $b \mapsto e_{1}=(1,0, \ldots, 0) \in S_{b}^{n-1} \subset \mathbb{R}^{n}$, where $S_{b}^{n-1}=\tilde{\pi}^{-1}(b)$ is the fiber of $\tilde{\pi}$ over $b \in c_{k}$. The preimage $\tilde{\pi}^{-1}\left(c_{k}\right)$ is thus a direct product $c_{k} \times S^{n-1}$. Moreover, it admits a cellular decomposition of the form

$$
c_{k} \times\left\{e_{1}\right\} \cup c_{k} \times D^{n-1} .
$$

Since for any $k$-cell $c_{k} \in K$, the distinguished point $e_{1}$ is given by the section $s$, we have that the boundary operator for $S_{1} B$ satisfies

$$
\partial\left(c_{k} \times\left\{e_{1}\right\}\right)=\left(\partial c_{k}\right) \times\left\{e_{1}\right\}
$$

Moreover, since the bundle $\pi$ and hence $\tilde{\pi}$ are trivial over the closure $\overline{c_{k}}$, we have that

$$
\partial\left(c_{k} \times\left\{D_{n-1}\right\}\right)=\sum_{i} \pm(-1)^{i} c_{k}^{i} \times\left\{D_{n-1}\right\}
$$


where $\partial c_{k}=\sum(-1)^{i} c_{k}^{i}$ is the boundary of $c_{k}$ with the induced orientation. We observe that since $\pi$ and hence $\tilde{\pi}$ are orientable, the sign can be chosen so that $\partial\left(c_{k} \times\left\{D_{n-1}\right\}\right)=\partial c_{k} \times\left\{D_{n-1}\right\}$. We conclude that the boundary operator is the same as for the direct product.

To prove the last statement, consider the cell $\left\{b_{0}\right\} \times D^{n-1}$, where $b_{0}$ is a vertex of $K$. We observe that this cell is not a boundary of $C_{1} \times D^{n-1}$ or $C_{n} \times\left\{e_{1}\right\}$ (here $C_{1}$ and $C_{n}$ are some 1 and $n$ chains in $K$, respectively). Indeed, $\partial C_{1}$ consists of an even number of points and the boundary $\partial\left(C_{n} \times\left\{e_{1}\right\}\right)$ is transverse to the fibers.

Remark A.3. From Lemma A.2 it follows that if $\left.\pi\right|_{S_{1} B}: S_{1} B \rightarrow B$ admits a global section, then the homology groups of $S_{1} B$ can be computed using a Künneth formula.

Example A.4. As an example, consider the Stiefel manifold $V_{k, 2}$. It can be viewed as the unit tangent bundle of $S^{k-1}$. It is known that the integer homology groups of $V_{2 k, 2}$ are the same as for the product $S^{2 k-1} \times S^{2 k-2}$; see [14, Section 3.D]. On the other hand, $V_{2 k, 2}$ is not homeomorphic to the product $S^{2 k-1} \times S^{2 k-2}$, unless $k=1,2$ or 4 [1] and [15]. We note that the integer homology groups of $V_{2 k+1,2}$ are different from the homology groups of $S^{2 k} \times S^{2 k-1}$; in this case there is no global section since the base $S^{2 k}$ is even-dimensional.

Let $e(E)$ denote the Euler number of $\pi: E \rightarrow N$. We are ready to prove the desired result (Theorem 3.6).

Theorem A.5. Let $N$ be a closed orientable n-manifold and $\pi: E \rightarrow N$ be an orientable rank $n$ vector bundle over $N$. Consider the function $H=K+V \circ \pi: E \rightarrow \mathbb{R}$ on $E$ and let $x_{1}, \ldots, x_{L} \in V^{-1}\left(h_{c}\right)$ be the non-degenerate global maxima of the function $V$ on $N$. Then the topology of $H$ changes when $H$ passes the critical value $h_{c}$ if one of the following conditions is satisfied

1) $L=1$ and the Euler number $e(E)$ is not equal to \pm 1 ;

2) $L=2$ and the Euler number $e(E)$ does not vanish;

3) $L>2$.

Proof. The case $L>2$ was considered earlier, so we only need to consider the cases $L=1$ and $L=2$.

Case $L=1$. Let $x_{\max }$ be the unique non-degenerate global maximum of $V$ and $h_{\max }=H\left(x_{\max }, 0\right)=V\left(x_{\max }\right)$. Fix a small number $\varepsilon>0$. Then the level sets $\Sigma_{ \pm}:=$ $H^{-1}\left(h_{\max } \pm \varepsilon\right)$ are regular. We observe that the level $\Sigma_{+}$is homeomorphic to the unit sphere bundle $S_{1} N$ of $E$. 
Let $T_{ \pm}=H^{-1}\left(h_{c} \pm \varepsilon\right) \backslash \pi^{-1}(U)$, where $U$ is a small open disk containing the maximum $x_{\max }$. Observe that the sets $T_{-}$and $T_{+}$are sphere bundles over $L \backslash U$, and that these sphere bundles are isomorphic through a radial projection. Similarly to the proof of Lemma 3.1, we have that

$$
\begin{aligned}
& \Sigma_{-} \cong T_{-} \cup_{f_{-}} S^{n-1} \times D^{n} \text { and } \\
& \Sigma_{+} \cong T_{+} \cup_{f_{+}} D^{n} \times S^{n-1},
\end{aligned}
$$

where the attaching maps for the respective boundary components are such that $f_{-}\left(\{x\} \times \partial D^{n}\right)$ is a fiber of the sphere bundle $\pi: T_{-} \rightarrow N \backslash U$ and $f_{+}$comes from the sphere bundle structure on $\Sigma_{+}$. In particular, $f_{+}\left(\{x\} \times S^{n-1}\right)$ is a fiber of $\pi: T_{+} \rightarrow N \backslash U$ and $f_{+}\left(\partial D^{n} \times\{y\}\right)$ is a section over $\partial U$.

Consider a part of the homology exact sequences of the pairs $\left(\Sigma_{ \pm}, T_{ \pm}\right)$:

$$
G \stackrel{\partial_{*}}{\longrightarrow} H_{n-1}\left(T_{ \pm} ; G\right) \longrightarrow H_{n-1}\left(\Sigma_{ \pm} ; G\right) \longrightarrow 0
$$

Observe that the map $H_{n-1}\left(T_{-} ; G\right) \rightarrow H_{n-1}\left(\Sigma_{-} ; G\right)$ has a non-trivial kernel, given by the homology class of the fiber $f_{-}\left(\{x\} \times \partial D^{n}\right)$; this homology class is non-trivial in $H_{n-1}\left(T_{-} ; G\right)$ by Lemma A.2. On the other hand, this map is surjective.

The bundle $\pi: T_{+} \rightarrow N \backslash U$ also has a global section. We denote it by $f$. We observe that the restriction of $f_{+}^{-1} \circ f$ to the boundary sphere $\partial U \cong S^{n-1}$ is a map of degree $k \neq \pm 1$, where $k$ is the Euler number. (Strictly speaking, $f_{+}^{-1} \circ f$ maps into $D^{n} \times S^{n-1}$, but this space deformation retracts onto $S^{n-1}$.) The section $\partial D^{n} \times\{y\}$ gives rise to a map of degree 0 .

Setting $G=\mathbb{Z}_{k}$ for $k \neq 0$ and $G=\mathbb{R}$ for $k=0$, we get that $f(\partial U)$ and $f_{+}\left(\partial D^{n} \times\right.$ $\{y\})$ are of the same $G$-homology class in $\partial T_{+}$. Hence $\left[f_{+}\left(\partial D^{n} \times\{y\}\right)\right]$ is trivial in the group $H_{n-1}\left(T_{+} ; G\right)$ and

$$
H_{n-1}\left(\Sigma_{+}, G\right) \cong H_{n-1}\left(T_{+} ; G\right)
$$

when $G=\mathbb{Z}_{k}$ (or $\mathbb{R}$ when $k=0$ ). Since $T_{-}$and $T_{+}$are diffeomorphic and also compact, it follows that the $(n-1)$-th homology groups $H_{n-1}\left(\Sigma_{+} ; G\right)$ and $H_{n-1}\left(\Sigma_{-} ; G\right)$ are not isomorphic for $G=\mathbb{Z}_{k}$.

Case $L=2$. We shall assume that the two maxima are on close, but different level sets of $V$, and that we are passing both of these maxima at the same time. Then one maximum becomes local and the other global.

Consider what happens when we pass the second (global) maximum. We observe that there is an almost global section $f$ such that the restriction of $f_{+}^{-1} \circ f$ to the boundary sphere $\partial U \cong S^{n-1}$ is a map of degree $k \neq \pm 1$, where $k$ is the Euler number. By the assumption, $k \neq 0$. Observe that $f(\partial U)$ is trivial in the group $H_{n-1}\left(T_{+} ; G\right)$. However, if $G=\mathbb{R}$, then $f_{+}\left(\partial D^{n} \times\{y\}\right)$ is non-trivial in $H_{n-1}\left(T_{+} ; G\right)$. 
Indeed, using a suitable cellular decomposition of $T_{+}$(see Lemma A.2), we get that any relative $n$-cycle in $T_{+}$is given by linear combinations of

a) The products of relative 1 -cycles in $(N \backslash U, \partial U)$ and $D^{n-1}$, where $D^{n-1} \cup$ $\{p t\}=S^{n-1}$

b) The section $f(N \backslash U)$.

But the boundary of any $n$-cycle as in a) vanishes in $\partial U \times S^{n-1}$, whereas $\partial f(N \backslash U)$ and $f_{+}\left(\partial D^{n} \times\{y\}\right)$ are different homology cycles in $\partial U \times S^{n-1}$ since $k \neq 0$. It follows that $f_{+}\left(\partial D^{n} \times\{y\}\right)$ is not a boundary and that

$$
b_{n-1}\left(\Sigma_{+}, \mathbb{R}\right)+1=b_{n-1}\left(T_{+}, \mathbb{R}\right) .
$$

Since

$$
b_{n-1}\left(\Sigma_{-}, \mathbb{R}\right)+1=b_{n-1}\left(T_{-}, \mathbb{R}\right)
$$

and since $T_{-}$and $T_{+}$are homeomorphic and also compact, we get that $b_{n-1}\left(\Sigma_{+}, \mathbb{R}\right)=$ $b_{n-1}\left(\Sigma_{-}, \mathbb{R}\right)$. But Lemma 3.1 implies that the other (local) maximum contributes to the change of the $(n-1)$-Betti number, also when $G=\mathbb{R}$. The result follows.

Remark A.6. Assume that the maxima $x_{1}, \ldots, x_{L}$ are not located on one critical level, but belong to (the interior of) the set $V^{-1}[a, b], a<b$, that contains no other critical points. In this case, the same result holds if one compares the topology of $H^{-1}(a)$ with that of $H^{-1}(b)$; cf. Subsection 4.1 .

Remark A.7. We note that if $L=1$ and the Euler class vanishes, then the $(n-1)$-Betti number changes according to

$$
b_{n-1}\left(H^{-1}\left(h_{c}+\varepsilon\right) ; \mathbb{F}\right)=b_{n-1}\left(H^{-1}\left(h_{c}-\varepsilon\right) ; \mathbb{F}\right)+1,
$$

where $\mathbb{F}$ is a field; cf. Lemma 3.1.

\section{References}

1. Adams, F. J., On the nonexistence of elements of Hopf invariant one, Ann. of Math. (2) 72 (1960), 20-104.

2. Albouy, A., Integral manifolds of the N-body problem, Invent. Math. 11 (1993), 463-488.

3. Bolsinov, A. V., Dullin, H. R. and Wittek, A., Topology of energy surfaces and existence of transversal Poincaré sections, J. Phys. A 29 (1996), 4977-4985.

4. Bolsinov, A. V. and Fomenko, A. T., Integrable Hamiltonian Systems: Geometry, Topology, Classification, CRC Press, 2004.

5. Bleiler, S. A., Hodgson, C. D. and Weeks, J. R., Cosmetic surgery on knots, Geom. Topol. Monogr. 2 (1999), 23-34.

6. Dold, A., Lectures on algebraic topology, Grundlehren der mathematischen Wissenschaften 200, Springer, 1995. 
7. Dubrovin, B. A., Fomenko, A. T. and Novikov, S. P., Modern Geometry-Methods and Applications: Part III: Introduction to Homology Theory, Graduate Texts in Mathematics 124, Springer, 1990.

8. Duistermate, H., On global action-angle coordinates, Comm. Pure Appl. Math. 33 (1980), 687-706.

9. Efstathiou, K., Metamorphoses of Hamiltonian Systems with Symmetries, Lecture Notes in Mathematics 1864, Springer, 2005.

10. Efstathiou, K. and Martynchuk, N., Monodromy of Hamiltonian Systems with Complexity-1 Torus Actions, J. Geom. Phys. 115 (2017), 104-115.

11. Fomenko, A. T. and Fuchs, D. B., Homotopical topology (Moscow University Press, 1969) 273, Springer, 2016.

12. Fomenko, A. T. and Zieschang, H., Topological invariant and a criterion for equivalence of integrable Hamiltonian systems with two degrees of freedom, Izv. Akad. Nauk SSSR Ser. Mat. 54 (1990), 546-575.

13. Gordon, C. M. and Luecke, J., Knots are determined by their complements, J. Amer. Math. Soc. 2 (1989), 371-415.

14. Hatcher, A., Algebraic Topology, Cambridge University Press, 2002.

15. James, I. M. and Whitehead, J. H. C., The Homotopy Theory of Sphere Bundles Over Spheres (I), Proc. London Math. Soc. s3-4 (1954), 196-218.

16. Kervaire, M. A. and Milnor, J. W., Groups of homotopy spheres: I, Ann. of Math. 77 (1963), 504-537.

17. Kirby, R. (ed.), Problems in low-dimensional topology, in: Geometric topology (Athens, GA 1993), AMS/IP Studies in Advanced Mathematics 2 (part 2), pp. 35-473, American Mathematical Society, Providence, 1997.

18. Konno, T., Unit Tangent Bundle over Two-Dimensional Real Projective Space, Nihonkai Math. J. 1 (2002), 57-66.

19. Martynchuk, N., Broer, H. W. and Efstathiou, K., Hamiltonian monodromy and Morse theory, Communications in Mathematical Physics (2019).

20. Martynchuk, N. and Efstathiou, K., Parallel Transport Along Seifert Manifolds and Fractional Monodromy, Comm. Math. Phys. 356 (2017), 427-449.

21. Clerk Maxwell, J., On Hills and Dales, Philos. Mag. 40 (1870), 269. 421-427.

22. MCCord, C., On the homology of the integral manifolds in the planar N-body problem, Ergodic Theory Dynam. Systems 21 (2001), 861-883.

23. McCord, C., Meyer, K. and Wang, Q., The integral manifolds of the three body problem, in Memoirs of the American Mathematical Society 628, American Mathematical Soc., 1998.

24. Milnor, J. W., Morse theory, Princeton University Press, Princeton, N. J., 1963.

25. Milnor, J. W., A procedure for killing the homotopy groups of differentiable manifolds, Proc. Sympos. Pure Math. 3 (1962).

26. Milnor, J. W., On Manifolds Homeomorphic to the 7-Sphere, Ann. of Math. 64 (1956), 399-405.

27. Nicolaescu, L., An Invitation to Morse Theory (Universitext), Springer, 2011.

28. Sheldon Palais, R. and Smale, S., A generalized Morse theory, Bull. Amer. Math. Soc. (N.S.) 70 (1964), 165-172.

29. Palmore, J., Measure of degenerate relative Equilibria. I, Ann. of Math. 104 (1976), $421-429$ 
30. Palmore, J., Classifying relative equilibria. I, Bull. Amer. Math. Soc. (N.S.) 79 (1973), 904-908.

31. RAnicki, A., Algebraic and Geometric Surgery, Oxford University Press, 2002.

32. Smale, S., Topology and mechanics. I, Invent. Math. 10 (1970), 305-331.

33. Smale, S., Topology and mechanics. II. The Planar n-Body Problem, Invent. Math. 11 (1970), 45-64.

Andreas Knauf

Department of Mathematics

Friedrich-Alexander-University Erlangen-

Nürnberg

Cauerstr. 11, D-91058

Erlangen

Germany

knauf@math.fau.de
Nikolay Martynchuk

Department of Mathematics

Friedrich-Alexander-University Erlangen-

Nürnberg

Cauerstr. 11, D-91058

Erlangen

Germany

and

Moscow Center for Fundamental and Applied Mathematics

Moscow

Russia

martynchuk@math.fau.de

Received March 11, 2020

in revised form July 16, 2020 